Available online at http://jnfa.mathres.org
MATHES Journal of Nonlinear Functional Analysis
A

\title{
PERIODIC MILD SOLUTIONS OF INFINITE DELAY EVOLUTION EQUATIONS WITH NON-INSTANTANEOUS IMPULSES
}

\author{
SAÏD ABBAS ${ }^{1}$, NASSIR AL ARIFI ${ }^{2}$, MOUFFAK BENCHOHRA ${ }^{3,4}$, JOHN GRAEF $^{5, *}$ \\ ${ }^{1}$ Laboratory of Mathematics, Geometry, Analysis, Control and Applications \\ Tahar Moulay University of Saïda, P.O. Box 138, EN-Nasr, 20000 Saïda, Algeria \\ ${ }^{2}$ College of Science, Geology and Geophysics Department, King Saud University, Riyadh 11451, Saudi Arabia \\ ${ }^{3}$ Laboratory of Mathematics, Djillali Liabes University of Sidi Bel-Abbès, \\ P.O. Box 89, Sidi Bel-Abbès 22000, Algeria \\ ${ }^{4}$ Department of Mathematics, College of Science, King Saud University \\ ${ }^{5}$ Department of Mathematics, University of Tennessee at Chattanooga, Chattanooga, TN 37403, USA
}

\begin{abstract}
This paper deals with the existence of periodic mild solutions for a class of functional evolution equations. The techniques used are a generalization of the classical Darbo fixed point theorem in Banach spaces. We show that the Poincaré operator is a condensing operator with respect to Kuratowski's measure of noncompactness in a determined phase space, and then derive periodic solutions from bounded solutions by using Sadovskii's fixed point theorem.
\end{abstract}

Keywords. Functional evolution equation; Evolution system; Densely defined operator; Periodic mild solution; Poincaré operator.

\section{INTRODUCTION}

There has been a significant development in functional evolution equations in recent years; see the monographs $[1,2,3,4]$ and the papers $[5,6,7]$. In [8], an iterative method was used for the existence of mild solutions of evolution equations and inclusions. Using Tichonov's fixed point theorem, Olszowy and Wȩdrychowicz [9] considered a class of evolution equations on unbounded intervals. However, in previous papers some restrictions like the compactness of the semigroup, Lipschitz conditions on the nonlinear term, or the boundedness of the obtained mild solutions, were assumed.

Most of the earlier research papers deal with the existence of solutions for differential equations with instantaneous impulsive conditions (see [10, 11, 12, 13, 14]). But many times it is

\footnotetext{
${ }^{*}$ Corresponding author.

E-mail addresses: abbasmsaid@yahoo.fr (S. Abbas), nalarifi@ksu.edu.sa (N. Al Arifi), benchohra@ yahoo.com (M. Benchohra), John-Graef@utc.edu (J. Graef).

Received July 30, 2019; Accepted February 9, 2020.
} 
known that certain dynamics of evolution processes cannot be describe by instantaneous impulses. For example, in pharmacotherapy, high or low levels of glucose can be interpreted as an impulsive action that start abruptly at a certain point in time and continue for a finite time interval. Such types of systems are known as non-instantaneous impulsive systems and these are more suitable in the study of the dynamics of evolution processes [15].

This theory of a new class of impulsive differential equation was initiated by Hernández et al. [16]. Pierri et al. [17] continued the work in this field and extended the theory of [16] to a $P C_{\alpha}$ normed Banach space. The existence of solutions for non-instantaneous impulsive differential equations had also been discussed in [18].

In this paper, we discuss the existence of periodic mild solutions to the class of functional differential equations with infinite delay and non-instantaneous impulses

$$
\left\{\begin{array}{l}
u^{\prime}(t)+A(t) u(t)=f\left(t, u(t), u_{t}\right), \text { if } t \in I_{k}, k=0,1, \ldots \\
u(t)=g_{k}\left(t, u\left(t_{k}^{-}\right)\right), \text {if } t \in J_{k}, k=1,2, \ldots \\
u(t)=\phi(t), \text { if } t \in \mathbb{R}_{-}:=(-\infty, 0]
\end{array}\right.
$$

where $I_{0}=\left[0, t_{1}\right], I_{k}:=\left(s_{k}, t_{k+1}\right], J_{k}:=\left(t_{k}, s_{k}\right], 0=s_{0}<t_{1} \leq s_{1} \leq t_{2}<\cdots<s_{m-1} \leq t_{m} \leq s_{m} \leq$ $t_{m+1}=T \leq s_{m+1} \leq t_{m+2} \leq \ldots<+\infty,\left(E,\|\cdot\|_{E}\right)$ is a real Banach space, $f: I_{k} \times E \times \mathscr{B} \rightarrow E$, $k=0, \ldots, g_{k}: J_{k} \times E \rightarrow E, k=1,2, \ldots$, are given functions $T$-periodic in $t, T>0, \mathscr{B}$ is an abstract phase space to be specified later, and $\phi: \mathbb{R}_{-} \rightarrow E$ is a given function. Here, $\{A(t)\}_{t>0}$ is a $T$-periodic family of unbounded operators from $E$ into $E$ that generate an evolution system of operators $\{U(t, s)\}_{(t, s) \in \mathbb{R}_{+} \times \mathbb{R}_{+}}$for $(t, s) \in \Lambda:=\left\{(t, s) \in \mathbb{R}_{+} \times \mathbb{R}_{+}: 0 \leq s \leq t<+\infty\right\}$, where $\mathbb{R}_{+}:=[0, \infty)$.

For any continuous function $u$ and any $t \in \mathbb{R}_{+}$, we denote by $u_{t}$ the element of $\mathscr{B}$ defined by $u_{t}(\theta)=u(t+\theta)$ for $\theta \in \mathbb{R}_{-}:=(-\infty, 0]$. Here, $u_{t}(\cdot)$ represents the history of the state up to the present time $t$. We assume that the histories $u_{t}$ belong to $\mathscr{B}$.

In $[19,20]$, the existence of periodic mild solution of delay evolution equations subject to instantaneous impulse was considered. In this paper, we consider the existence of periodic mild solutions for evolution equations with infinite delay and non-instantaneous impulses, which are a more general class of impulsive evolution equations. We use the classical Darbo fixed point theorem, the Poincaré operator, and the concept of a measure of noncompactness in Banach spaces.

The paper is organized as follows. In Section 2, some preliminary results are introduced. The main results is presented in Section 3, while the last section, Section 4 is devoted to an illustrative example.

\section{PRELIMINARIES}

Let $I:=[0, T], T>0$. A measurable function $u: I \rightarrow E$ is Bochner integrable if and only if $\|u\|$ is Lebesgue integrable. For properties of the Bochner integral, see, for instance, Yosida [21].

By $B(E)$ we mean the Banach space of all bounded linear operators from $E$ into $E$ with the norm

$$
\|N\|_{B(E)}=\sup _{\|u\|=1}\|N(u)\|
$$


As usual, $L^{1}(I, E)$ denotes the Banach space of measurable functions $u: I \rightarrow E$ that are Bochner integrable and normed by

$$
\|u\|_{L^{1}}=\int_{0}^{T}\|u(t)\| d t
$$

By $\mathscr{C}:=C(I)$ we denote the Banach space of all continuous functions from $I$ into $E$ with the norm $\|\cdot\|_{\infty}$ defined by

$$
\|u\|_{\infty}=\sup _{t \in I}\|u(t)\|
$$

We consider the space

$$
\begin{array}{r}
\tilde{C}((-\infty, 0], E)=\left\{u:(-\infty, 0] \rightarrow E \mid u \text { is continuous and there exist } \tau_{k} \in(-\infty, 0),\right. \\
\left.k=1, \ldots, m, \text { such that } u\left(\tau_{k}^{-}\right) \text {and } u\left(\tau_{k}^{+}\right) \text {exist with } u\left(\tau_{k}^{-}\right)=u\left(\tau_{k}\right)\right\},
\end{array}
$$

and the Banach space

$$
\begin{aligned}
P C= & \left\{u:(-\infty, T] \rightarrow E|u|_{\mathbb{R}_{-}} \in \mathscr{B},\left.u\right|_{J_{k}}=g_{k}, k=1, \ldots, m,\left.u\right|_{I_{k}}, k=1, \ldots, m,\right. \\
& \text { is continuous, and } u\left(s_{k}^{-}\right), u\left(s_{k}^{+}\right), u\left(t_{k}^{-}\right) \text {and } u\left(t_{k}^{+}\right) \text {exist } \\
& \text { with } \left.u\left(s_{k}^{+}\right)=g_{k}\left(s_{k}, u\left(s_{k}^{-}\right)\right) \text {and } u\left(t_{k}^{-}\right)=g_{k}\left(t_{k}, u\left(t_{k}^{-}\right)\right)\right\},
\end{aligned}
$$

with the norm

$$
\|u\|_{P C}=\max \left\{\|u\|_{\infty},\|\phi\|_{\mathscr{B}}\right\} .
$$

In what follows, for the family $\{A(t), t \geq 0\}$ of closed densely defined linear unbounded operators on Banach space $E$, we assume that it satisfies the following assumptions (see [2], p. 158).

$\left(P_{1}\right)$ The domain $D(A(t))$ is independent of $t$ and is dense in $E$.

$\left(P_{2}\right)$ For $t \geq 0$, the resolvent $R(\lambda, A(t))=(\lambda I-A(t))^{-1}$ exists for all $\lambda$ with $\operatorname{Re} \lambda \leq 0$, and there is a constant $K$ independent of $\lambda$ and $t$ such that

$$
\|R(t, A(t))\| \leq K(1+|\lambda|)^{-1}, \text { for } \operatorname{Re} \lambda \leq 0 .
$$

$\left(P_{3}\right)$ There exist constants $L>0$ and $0<\alpha \leq 1$ such that

$$
\left\|(A(t)-A(\theta)) A^{-1}(\tau)\right\| \leq L|t-\tau|^{\alpha}, \text { for } t, \theta, \tau \in I .
$$

Lemma 2.1. ([2, 22]) Under assumptions $\left(P_{1}\right)-\left(P_{3}\right)$, the Cauchy problem

$$
u^{\prime}(t)-A(t) u(t)=0, t \in I, u(0)=y_{0},
$$

has a unique evolution system $U(t, s),(t, s) \in \Delta:=\{(t, s) \in J \times J: 0 \leq s \leq t \leq T\}$ satisfying the following properties:

(1) $U(t, t)=I$ where I is the identity operator in $E$;

(2) $U(t, s) U(s, \tau)=U(t, \tau)$ for $0 \leq \tau \leq s \leq t \leq T$;

(3) $U(t, s) \in B(E)$ the space of bounded linear operators on $E$, where for every $(t, s) \in \Delta$ and for each $u \in E$, the mapping $(t, s) \rightarrow U(t, s) u$ is continuous.

More details on evolution systems and their properties can be found in the books of Ahmed [2] and Pazy [22].

In this paper, we assume that the state space $\left(\mathscr{B},\|\cdot\|_{\mathscr{B}}\right)$ is a seminormed linear space of functions mapping $\mathbb{R}_{-}$into $E$ that satisfy the following fundamental axioms introduced by Hale and Kato in [23]. 
$\left(A_{1}\right)$ : If $u \in P C$ and $u_{0} \in \mathscr{B}$, then, for every $t \in I$, the following conditions hold:

(i) $u_{t} \in \mathscr{B}$;

(ii) $\left\|u_{t}\right\|_{\mathscr{B}} \leq K(t) \int_{0}^{t}\|u(s)\| d s+M(t)\|\phi\|_{\mathscr{B}}$;

(iii) $\|u(t)\| \leq H\left\|u_{t}\right\|_{\mathscr{B}}$, where $H \geq 0$ is a constant, $K: I \rightarrow \mathbb{R}_{+}$is continuous, $M: \mathbb{R}_{+} \rightarrow$ $\mathbb{R}_{+}$is locally bounded, and $H, K, M$, are independent of $u(\cdot)$.

$\left(A_{2}\right)$ : For the function $u(\cdot)$ in $\left(A_{1}\right), u_{t}$ is a $\mathscr{B}$-valued continuous function on $I$.

$\left(A_{3}\right)$ : The space $\mathscr{B}$ is complete.

Denote

$$
K_{T}=\sup \{K(t): t \in I\} \text { and } M_{T}=\sup \{M(t): t \in I\} .
$$

Remark 2.2. Axiom $\left(A_{1}\right)$ (ii) is equivalent to $\|\phi(0)\| \leq H\|\phi\|_{\mathscr{B}}$, for every $\phi \in \mathscr{B}$. From this equivalence, we can see that for all $\phi, \psi \in \mathscr{B}$ such that $\|\phi-\psi\|_{\mathscr{B}}=0, \phi(0)=\psi(0)$.

Lemma 2.3. (Lemma 2.1 in [20]) There exists an integer $k_{0}>1$ such that

$$
\left(\frac{1}{2}\right)^{k_{0}-1} M<1
$$

where $M=\sup _{(t, s) \in \Delta}\|U(t, s)\|_{B(E)}$ is finite, and there exists a function $h$ on $(-\infty, 0]$ such that $h(0)=$ $1, h(-\infty)=+\infty, h$ is decreasing on $(-\infty, 0]$, and for $d \geq w_{0}:=\frac{T}{K_{0}}$, we have $\sup _{s \in(-\infty, 0]} \frac{h(s)}{h(s-d)} \leq$ $\frac{1}{2}$

Next we give two examples of phase spaces.

Example 2.4. For the function $h$ given in Lemma 2.3, we define the spaces $C_{h}:=\{\phi \in$ $\tilde{C}\left(\mathbb{R}_{-}, E\right): \frac{\phi(\theta)}{h(\theta)}$ is bounded on $\left.\mathbb{R}_{-}\right\}$and $C_{h}^{0}:=\left\{\phi \in C_{h}: \lim _{\theta \rightarrow-\infty} \frac{\phi(\theta)}{h(\theta)}=0\right\}$, endowed with the uniform norm

$$
\|\phi\|=\sup \left\{\frac{\|\phi(\theta)\|}{h(\theta)}: \theta \leq 0\right\} .
$$

Then the spaces $C_{h}$ and $C_{h}^{0}$ satisfy condition $\left(A_{3}\right)$. Also, $C_{h}$ and $C_{h}^{0}$ satisfy conditions $\left(A_{1}\right)$ and $\left(A_{2}\right)$ if

$$
\sup _{t \in I} \sup _{-\infty<\theta \leq-t} \frac{\phi(t+\theta)}{h(\theta)}<\infty .
$$

Example 2.5. For any real positive constant $\gamma$, we define the space

$$
\left.C_{\gamma}:=\{\phi \in \tilde{C}((-\infty, 0]), E): \lim _{\theta \rightarrow-\infty} e^{\gamma \theta} \phi(\theta) \text { exist in } E\right\}
$$

endowed with the norm

$$
\|\phi\|=\sup \left\{e^{\gamma \theta}\|\phi(\theta)\|: \theta \leq 0\right\} .
$$

Then in the space $C_{\gamma}$ the axioms $\left(A_{1}\right)-\left(A_{3}\right)$ are satisfied.

In all that follows, we consider the phase space

$$
\left.\mathscr{B}:=\{\phi \in \tilde{C}((-\infty, 0]), E): \sup _{s \in(-\infty, 0]} \frac{\|\phi(s)\|}{h(s)}<\infty\right\},
$$


where $h: \mathbb{R}_{-} \rightarrow \mathbb{R}_{+}$is the function given in Lemma 2.3. Then $\mathscr{B}$ endowed with the norm

$$
\|\phi\|_{\mathscr{B}}=\sup _{s \in(-\infty, 0]} \frac{\|\phi(s)\|}{h(s)}
$$

is a Banach space [24].

Next, we define the Kuratowski measure of noncompactness and give some of its basic properties.

Definition 2.6. ([25, 26]) Let $X$ be a Banach space and let $\Omega_{X}$ be the set of all bounded subsets of $X$. The Kuratowski measure of noncompactness is the map $\alpha: \Omega_{X} \rightarrow[0, \infty]$ defined by

$$
\alpha(B)=\inf \left\{\varepsilon>0: B \subseteq \cup_{i=1}^{n} B_{i} \text { and } \operatorname{diam}\left(B_{i}\right) \leq \varepsilon\right\}
$$

where $B \in \Omega_{E}$ and

$$
\operatorname{diam}\left(B_{i}\right)=\sup \left\{\|u-v\|_{E}: u, v \in B_{i}\right\} .
$$

The Kuratowski measure of noncompactness satisfies the following properties.

Lemma 2.7. ([25, 27]) Let $A$ and $B$ be bounded sets.

(a) $\alpha(B)=0$ if and only if $\bar{B}$ is compact (i.e., $B$ is relatively compact), where $\bar{B}$ denotes the closure of $B$.

(b) nonsingularity: $\alpha$ is equal to zero on every one element set.

(c) If $B$ is a finite set, then $\alpha(B)=0$.

(d) $\alpha(B)=\alpha(\bar{B})=\alpha(\operatorname{conv} B)$, where conv $B$ is the convex hull of $B$.

(e) monotonicity: $A \subset B$ implies $\alpha(A) \leq \alpha(B)$.

(f) algebraic semi-additivity: $\alpha(A+B) \leq \alpha(A)+\alpha(B)$, where $A+B=\{x+y: x \in A, y \in$ $B\}$.

(g) semi-homogencity: $\alpha(\lambda B)=|\lambda| \alpha(B), \lambda \in \mathbb{R}$, where $\lambda(B)=\{\lambda x: x \in B\}$.

(h) semi-additivity: $\alpha(A \cup B)=\max \{\alpha(A), \alpha(B)\}$.

(i) $\alpha(A \cap B)=\min \{\alpha(A), \alpha(B)\}$.

(j) invariance under translations: $\alpha\left(B+x_{0}\right)=\alpha(B)$ for any $x_{0} \in X$.

Lemma 2.8. ([28]) Let $V \subset C(I, E)$ be a bounded and equicontinuous set. Then

(i) the function $t \rightarrow \alpha(V(t))$ is continuous on I, and

$$
\alpha_{c}(V)=\sup _{t \in I} \alpha(V(t)) .
$$

(ii) $\alpha\left(\int_{0}^{T} u(s) d s: u \in V\right) \leq \int_{0}^{T} \alpha(V(s)) d s$,

where

$$
V(t)=\{u(t): u \in V\}, t \in I .
$$

Lemma 2.9. ([29]) If $Y$ is a bounded subset of a Banach space $\mathscr{X}$, then, for each $\varepsilon>0$, there is a sequence $\left\{y_{k}\right\}_{k=1}^{\infty} \subset Y$ such that

$$
\alpha(Y) \leq 2 \alpha\left(\left\{y_{k}\right\}_{k=1}^{\infty}\right)+\varepsilon .
$$

Lemma 2.10. ([30]) If $\left\{u_{k}\right\}_{k=1}^{\infty} \subset L^{1}(I)$ is uniformly integrable, then $\alpha\left(\left\{u_{k}\right\}_{k=1}^{\infty}\right)$ is measurable and

$$
\alpha\left(\left\{\int_{0}^{t} u_{k}(s) d s\right\}_{k=1}^{\infty}\right) \leq 2 \int_{0}^{t} \alpha\left(\left\{u_{k}(s)\right\}_{k=1}^{\infty}\right) d s .
$$


For our purposes here, we will need the following fixed point theorem.

Theorem 2.11. (Darbo's Fixed Point Theorem, [31,32]) Let $X$ be a Banach space and $C$ be a bounded, closed, convex, and nonempty subset of $X$. Let $N: C \rightarrow C$ be a continuous mapping such that for all closed subsets $D$ of $C$,

$$
\alpha(T(D)) \leq k \alpha(D),
$$

where $0 \leq k<1$ and $\alpha$ is the Kuratowski measure of noncompactness. Then $T$ has a fixed point in $C$.

Remark 2.12. Mappings satisfying the Darbo-condition (2.1) have subsequently been called $\mathrm{k}$-set contractions.

Definition 2.13. Let $X$ be a Banach space and $\alpha$ be a measure of noncompactness. An operator $P: X \rightarrow X$ is said to be condensing if $P$ is continuous, takes bounded sets into bounded sets, and $\alpha(P(B)) \leq \alpha(B)$ for every bounded set $B \subset X$ with $\alpha(B)>0$.

Theorem 2.14. (Sadovskii's fixed point theorem, [20]) Let $X$ be a Banach space, $\alpha$ be a measure of noncompactness, and $P: X \rightarrow X$ be a condensing operator. If $P(H) \subset H$ for a convex, closed, and bounded set $H$ of $X$, then $P$ has a fixed point in $H$.

\section{Periodic Mild Solutions}

Definition 3.1. By a periodic mild solution of problem (1.1), we mean a measurable and Tperiodic function $u$ that satisfies

$$
u(t)=\left\{\begin{array}{l}
U(t, 0) \phi(0)+\int_{0}^{t} U(t, s) f\left(s, u(s), u_{s}\right) d s, \text { if } t \in I_{0}, \\
U\left(t, s_{k}\right) g_{k}\left(s_{k}, u\left(s_{k}^{-}\right)\right)+\int_{s_{k}}^{t} U(t, s) f\left(s, u(s), u_{s}\right) d s, \text { if } t \in I_{k}, k=1, \ldots, m, \\
g_{k}\left(t, u\left(t_{k}^{-}\right)\right), \text {if } t \in J_{k}, k=1, \ldots, m, \\
\phi(t), \text { if } t \in \mathbb{R}_{-} .
\end{array}\right.
$$

The following hypotheses will be used in the sequel.

$\left(H_{1}\right)$ The functions $f$ and $g_{k}$ are continuous and they map bounded sets into bounded sets.

$\left(H_{2}\right)$ The function $t \mapsto f(t, u, v)$ is measurable on $I_{k}, k=0, \ldots, m$, for each $u, v \in E \times \mathscr{B}$, and the functions $u \mapsto f(t, u, v)$ and $v \mapsto f(t, u, v)$ are continuous on $E \times \mathscr{B}$ for a.e. $t \in I_{k}$, $k=0, \ldots, m$.

$\left(H_{3}\right)$ There is a constant $T>0$ such that $f(t+T, u, v)=f(t, u, v), A(t+T)=A(t), t \in I_{k}$, $k=0, \ldots, m, u, v \in E \times \mathscr{B}$, and $g_{k}(t+T, z)=g_{k}(t, z), t \in J_{k}, k=1, \ldots, m, z \in E$.

$\left(H_{4}\right)$ There exist continuous functions $p: I_{k} \rightarrow \mathbb{R}_{+}$and $q: J_{k} \rightarrow \mathbb{R}_{+}$such that

$$
|| f(t, u, v) \mid \leq p(t), \text { for a.e. } t \in I_{k}, k=0, \ldots, m \text {, and each } u, v \in E \times \mathscr{B},
$$

and

$$
|| g_{k}(t, z) \mid \leq q(t) \text {, for a.e. } t \in J_{k} \text {, and each } z \in E, k=0, \ldots, m .
$$

$\left(H_{5}\right)$ For bounded and measurable sets $B(t) \subset E, t \in \mathbb{R}_{+}$, and $B_{t} \subset \mathscr{B}, t \in \mathbb{R}_{+}$, such that

$$
B(t)=\{u(t): u \in C(I)\} \text { and } B_{t}=\left\{u_{t}: u_{t} \in \mathscr{B}\right\},
$$


we have

$$
\alpha\left(f\left(t, B(t), B_{t}\right)\right) \leq p(t) \alpha(B) \text { for a.e. } t \in I_{k}, k=0, \ldots, m,
$$

and

$$
\alpha\left(g_{k}(t, B)\right) \leq q(t) \alpha(B) \text { for a.e. } t \in J_{k}, k=1, \ldots, m,
$$

where $\alpha$ is a measure of noncompactness on the Banach space $E$.

Set

$$
M=\sup _{(t, s) \in \Delta}\|U(t, s)\|_{B(E)}, p^{*}=\sup _{t \in I_{k}} p(t), \text { and } q^{*}=\sup _{t \in J_{k}} q(t) .
$$

We shall prove our main result on the existence of periodic mild solutions to problem (1.1).

Theorem 3.2. Assume that conditions $\left(H_{1}\right)-\left(H_{5}\right)$ hold. If $4 M T p^{*}<1$, then problem (1.1) has at least one T-periodic mild solution defined on $\mathbb{R}$.

Proof. The proof will be given in two parts; we will first show the existence of a mild solution and then show that it is periodic. Consider the problem

$$
\left\{\begin{array}{l}
u^{\prime}(t)+A(t) u(t)=f\left(t, u(t), u_{t}\right), \text { if } t \in I_{k}, k=0, \ldots, m, \\
u(t)=g_{k}\left(t, u\left(t_{k}^{-}\right)\right), \text {if } t \in J_{k}, k=1, \ldots, m, \\
u(t)=\phi(t), \text { if } t \in \mathbb{R}_{-}:=(-\infty, 0],
\end{array}\right.
$$

Part 1. We begin by showing that (3.1) has a mild solution $u \in P C$ with $\|u\|_{P C} \leq R$, where

$$
R \geq \max \left\{\|\phi\|_{\mathscr{B}}, q^{*}, M\left(\|\phi(0)\|+p^{*}\right), M\left(q^{*}+p^{*}\right)\right\} .
$$

Consider the operator $N: P C \rightarrow P C$ defined by

$$
(N u)(t)=\left\{\begin{array}{l}
U(t, 0) \phi(0)+\int_{0}^{t} U(t, s) f\left(s, u(s), u_{s}\right) d s, \text { if } t \in I_{0}, \\
U\left(t, s_{k}\right) g_{k}\left(s_{k}, u\left(s_{k}^{-}\right)\right)+\int_{s_{k}}^{t} U(t, s) f\left(s, u(s), u_{s}\right) d s, \text { if } t \in I_{k}, k=1, \ldots, m, \\
g_{k}\left(t, u\left(t_{k}^{-}\right)\right), \text {if } t \in J_{k}, k=1, \ldots, m, \\
\phi(t), \text { if } t \in \mathbb{R}_{-} .
\end{array}\right.
$$

Clearly, the fixed points of the operator $N$ are mild solutions of problem (3.1).

For any $u \in P C$ and each $t \in I_{0}$, we have

$$
\begin{aligned}
\|(N u)(t)\| & \leq M\|\phi(0)\|+M \int_{0}^{t}\left\|f\left(s, u(s), u_{s}\right)\right\| d s \\
& \leq M\|\phi(0)\|+M p^{*} \leq R .
\end{aligned}
$$

Next, for any $u \in P C$ and each $t \in I_{k}, k=1, \ldots, m$, we have

$$
\begin{aligned}
\|(N u)(t)\| & \leq M q^{*}+M \int_{s_{k}}^{t}\left\|f\left(s, u(s), u_{s}\right)\right\| d s \\
& \leq M q^{*}+M p^{*} \leq R .
\end{aligned}
$$

Also, for any $u \in P C$ and each $t \in J_{k}, k=1, \ldots, m$, we have

$$
\|(N u)(t)\| \leq q^{*} \leq R
$$


and for any $u \in P C$ and each $t \in \mathbb{R}_{-}$, we have

$$
\|(N u)(t)\|=\|\phi\|_{\mathscr{B}} \leq R .
$$

This proves that $N$ transforms the ball $B_{R}:=\left\{w \in P C:\|w\|_{P C} \leq R\right\}$ into itself. We shall now show that the operator $N: B_{R} \rightarrow B_{R}$ satisfies all the assumptions of Theorem 2.11. The proof will be given in two steps.

Step 1. $N: B_{R} \rightarrow B_{R}$ is continuous.

Let $\left\{u_{n}\right\}_{n \in \mathbb{N}}$ be a sequence such that $u_{n} \rightarrow u$ in $B_{R}$. For each $t \in \mathbb{R}_{-} \cup J_{k}, k=1, \ldots, m$, we have

$$
\left\|\left(N u_{n}\right)(t)-(N u)(t)\right\|=0 \rightarrow 0 \quad \text { as } n \rightarrow \infty,
$$

and, for each $t \in I_{k}, k=0, \ldots, m$, we have

$$
\left\|\left(N u_{n}\right)(t)-(N u)(t)\right\| \leq M \int_{0}^{t}\left\|f\left(s, u_{n}(s), u_{s n}\right)-f\left(s, u(s), u_{s}\right)\right\| d s .
$$

Since $u_{n} \rightarrow u$ as $\mathrm{n} \rightarrow \infty$ and $f$ is continuous, by the Lebesgue dominated convergence theorem, inequality (3.3) implies

$$
\left\|\left(N u_{n}\right)(t)-(N u)(t)\right\| \rightarrow 0 \quad \text { as } n \rightarrow \infty .
$$

Hence,

$$
\left\|N\left(u_{n}\right)-N(u)\right\|_{P C} \rightarrow 0 \quad \text { as } n \rightarrow \infty .
$$

Step 2. For each closed subset $D$ of $C(I), \alpha(N(D)) \leq \ell \alpha(D)$.

From Lemmas 2.9 and 2.10, for any $D \subset B_{R}$ and any $\varepsilon>0$, there exists a sequence $\left\{u_{k}\right\}_{k=0}^{\infty} \subset$ $D$, such that, for all $t \in I_{k}, k=0, \ldots, m$,

$$
\begin{aligned}
\alpha((N D)(t)) & =\alpha\left(\left\{U(t, 0) \phi(0)+\int_{0}^{t} U(t, s) f\left(s, u(s), u_{s}\right) d s ; u \in D\right\}\right) \\
& \leq 2 \alpha\left(\left\{\int_{0}^{t} U(t, s) f\left(s, u_{k}(s), u_{k s}\right) d s\right\}_{k=1}^{\infty}\right)+\varepsilon \\
& \leq 4 \int_{0}^{t} \alpha\left(\|U(t, s)\|_{B(E)}\left\{f\left(s, u_{k}(s), u_{k s}\right)\right\}_{k=1}^{\infty}\right) d s+\varepsilon \\
& \leq 4 M \int_{0}^{t} \alpha\left(\left\{f\left(s, u_{k}(s), u_{k s}\right)\right\}_{k=1}^{\infty}\right) d s+\varepsilon \\
& \leq 4 M \int_{0}^{t} p(s) \alpha\left(\left\{u_{k}(s)\right\}_{k=1}^{\infty}\right) d s+\varepsilon \\
& \leq 4 M p^{*} \int_{0}^{t} \alpha\left(\left\{u_{k}(s)\right\}_{k=1}^{\infty}\right) d s+\varepsilon \\
& \leq 4 M T p^{*} \alpha(D)+\varepsilon .
\end{aligned}
$$

For all $t \in I_{k}, k=1, \ldots, m$, we have

$$
\begin{aligned}
\alpha((N D)(t)) & =\alpha\left(\left\{U\left(t, s_{k}\right) g_{k}\left(s_{k}, u\left(s_{k}^{-}\right)\right)+\int_{s_{k}}^{t} U(t, s) f\left(s, u(s), u_{s}\right) d s ; u \in D\right\}\right) \\
& \leq 2 \alpha\left(\left\{\int_{0}^{t} U(t, s) f\left(s, u_{k}(s), u_{k s}\right) d s\right\}_{k=1}^{\infty}\right)+\varepsilon \\
& \leq 4 M T p^{*} \alpha(D)+\varepsilon .
\end{aligned}
$$


Since $\varepsilon>0$ is arbitrary, we have

$$
\alpha((N D)(t)) \leq 4 M T p^{*} \alpha(D)
$$

As a consequence of these two steps together with Theorem 2.11, we can conclude that $N$ has a fixed point in $u \in B_{R}$ that in turn is a mild solution of problem (1.1).

Part 2. Periodic mild solutions.

A standard approach in deriving $T$-periodic solutions is to define the Poincaré operator $P: \mathscr{B} \rightarrow \mathscr{B}$ given by $P(\phi)=u_{T}(\phi)$ such that

$$
(P \phi)(s)=u_{T}(s, \phi)=u(T+s, \phi), s \in \mathbb{R}_{-},
$$

which maps an initial function (or value) $\phi$ along the unique mild solution $u(\phi)$ to our problem (1.1) by $T$ - units (i.e., $T$ units along the unique solution $u(\cdot, \phi)$ determined by the initial function $\phi)$. We shall show that $P$ is a condensing operator with respect to Kuratowski's measure of non-compactness in the phase space $\mathscr{B}$ and that the other conditions of Theorem 2.14 hold. We do this in two steps.

Step 1. The fixed points of $P$ give rise to periodic mild solutions of (1.1).

Let $\phi \in \mathscr{B}$ be such that $p(\phi)=\phi$. Then for the solution $u(\cdot)=u(\cdot, \phi)$ with $u_{0}(\cdot, \phi)=\phi$, we can define $v(t)=u(t+T)$. Now, for $t>0$, we can use known properties of $U(t, s)$ in Lemma 2.1, and the fact that $A, f$, and $g_{k}$ are $T$-periodic in $t$ to obtain that $v$ is also a solution with $v_{0}(\cdot, \phi)=u_{T}(\phi)=u(\cdot, \phi)$. That is, we can obtain that

$$
v(t)=\left\{\begin{array}{l}
U(t, 0) \phi(0)+\int_{0}^{t} U(t, s) f\left(s, v(s), v_{s}\right) d s, \text { if } t \in I_{0}, \\
U\left(t, s_{k}\right) g_{k}\left(s_{k}, v\left(s_{k}^{-}\right)\right)+\int_{s_{k}}^{t} U(t, s) f\left(s, v(s), v_{s}\right) d s, \text { if } t \in I_{k}, k=1, \ldots, m \\
g_{k}\left(t, v\left(t_{k}^{-}\right)\right), \text {if } t \in J_{k}, k=1, \ldots, m, \\
\phi(t), \text { if } t \in \mathbb{R}_{-} .
\end{array}\right.
$$

Then, the uniqueness of $\{U(t, s)\}_{(t, s) \in \Lambda}$ implies that $v(t)=u(t)$, so $u(t)=u(t+T)$ is a $T$ periodic solution.

Step 2. $P$ is condensing.

To prove that the operator $P: \mathscr{B} \rightarrow \mathscr{B}$ is condensing, let $D \subset \mathscr{B}$ be bounded with $\alpha(D)>0$. From Theorem 4.1 in [20], we obtain

$$
\alpha(P(D)) \leq\left(\frac{1}{2}\right)^{k_{0}-1} M \alpha(D)<\alpha(D)
$$

Thus, from Theorem 2.14, $P$ has a fixed point that gives rise to a periodic mild solution of problem (1.1). 


\section{AN EXAMPLE}

Consider the functional evolution problem

$$
\begin{cases}\frac{\partial z}{\partial t}(t, x)=a(t, x) \frac{\partial^{2} z}{\partial x^{2}}(t, x)+Q\left(t, z(t, x), z_{t}(\cdot, x)\right), & x \in[0, \pi], t \in I_{k}, k=0, \cdots, \\ z(t, x)=g_{k}(t, x), & t \in \mathbb{R}_{+}, x \in[0, \pi], t \in J_{k}, k=1, \cdots, \\ z(t, 0)=z(t, \pi)=0, & t \in \mathbb{R}_{+}, \\ z(0, x)=\Phi(x), & x \in[0, \pi], \\ z(t, x)=\phi(t, x), & t \in \mathbb{R}_{-}, x \in[0, \pi],\end{cases}
$$

where $a(t, x): \mathbb{R}_{+} \times[0, \pi] \rightarrow \mathbb{R}$ is a continuous function and is uniformly Hölder continuous in $t, Q: \mathbb{R}_{+} \times \mathbb{R} \times C_{h} \rightarrow \mathbb{R}, \Phi:[0, \pi] \rightarrow \mathbb{R}$, and $\phi: \mathbb{R}_{-} \times[0, \pi] \rightarrow \mathbb{R}$ are continuous functions such that $\Phi(x)=\phi(0, x), x \in[0, \pi]$, and $C_{h}$ is the phase space defined in Example 2.4.

Consider $E=L^{2}([0, \pi], \mathbb{R})$ and define $A(t)$ by $A(t) w=a(t, x) w^{\prime \prime}$ with domain

$$
D(A)=\left\{w \in E: w, w^{\prime} \text { are absolutely continuous, } w^{\prime \prime} \in E, w(0)=w(\pi)=0\right\} .
$$

Then $A(t)$ generates an evolution system $U(t, s)$ (see [33]).

For $x \in[0, \pi]$, we have

$$
\begin{gathered}
y(t)(x)=z(t, x), \quad t \in \mathbb{R}_{+}, \\
f\left(t, u(t), u_{t}, x\right)=Q\left(t, z(t, x), z_{t}(\cdot, x)\right), \quad t \in \mathbb{R}_{+}, \\
u_{0}(x)=\Phi(t, x), \quad x \in[0, \pi],
\end{gathered}
$$

and

$$
u(t, x)=\phi(t, x), x \in[0, \pi] .
$$

Thus, under the above definitions of $f, u_{0}$, and $A$, system (4.1) can be represented by the functional evolution problem (1.1). Furthermore, additional conditions on $Q$ ensure that conditions $\left(H_{1}\right)-\left(H_{5}\right)$ hold. Consequently, Theorem 3.2 implies that the evolution problem (4.1) has at least one periodic mild solution.

\section{Acknowledgements}

The second and third authors extend their appreciation to Distinguished Scientist Fellowship Program (DSFP) at King Saud University (Saudi Arabia). The fourth author's research was supported in part by a University of Tennessee at Chattanooga SimCenter - Center of Excellence in Applied Computational Science and Engineering (CEACSE) grant.

\section{REFERENCES}

[1] S. Abbas, M. Benchohra, Advanced Functional Evolution Equations and Inclusions, Developments in Mathematics, 39, Springer, Cham, 2015.

[2] N. U. Ahmed, Semigroup Theory with Applications to Systems and Control, Pitman Research Notes in Mathematics Series, 246, Longman Scientific \& Technical, Harlow, Wiley, New York, 1991.

[3] S. Heikkila, V. Lakshmikantham, Monotone Iterative Technique for Nonlinear Discontinuous Differential Equations, Dekker, New York, 1994.

[4] J. Wu, Theory and Applications of Partial Functional Differential Equations, Applied Mathematical Sciences 119, Springer-Verlag, New York, 1996.

[5] S. Baghli, M. Benchohra, Global uniqueness results for partial functional and neutral functional evolution equations with infinite delay, Differential Integral Equations 23 (2010), 31-50. 
[6] S. Baghli, M. Benchohra, Multivalued evolution equations with infinite delay in Fréchet spaces, Electron. J. Qual. Theory Differ. Equ. 2008 (2008), No. 33.

[7] M. Frigon, A. Granas, Résultats de type Leray-Schauder pour des contractions sur des espaces de Fréchet, Ann. Sci. Math. Québec 22 (1998), 161-168.

[8] S. Abbas, W. Albarakati, M. Benchohra, Successive approximations for functional evolution equations and inclusions, J. Nonlinear Funct. Anal. 2017 (2017), Article ID 39.

[9] L. Olszowy, S. Wẹdrychowicz, Mild solutions of semilinear evolution equation on an unbounded interval and their applications, Nonlinear Anal. 72 (2010), 2119-2126.

[10] R.P. Agarwal, M. Benchohra, B.A. Slimani, Existence results for differential equations with fractional order impulses, Mem. Differential Equations. Math. Phys. 44 (2008), 1-21.

[11] K. Balachandran, S. Kiruthika, Existence of solutions of abstract fractional impulsive semilinear evolution equations, Electron. J. Qual. Theory Differ. Equ. 2010 (2010), No. 4.

[12] M. Benchohra, J. Henderson, S. K. Ntouyas, Impulsive Differential Equations and Inclusions, Contemporary Mathematics and Its Applications, Vol. 2, Hindawi New York, 2006.

[13] V. Lakshmikantham, D.D. Bainov, P.S. Simeonov, Theory of Impulsive Differential Equations, World Scientific, NJ, 1989.

[14] M. Meghnafi, M. Benchohra, K. Aissani, Impulsive fractional evolution equations with state-dependent delay, Nonlinear Stud. 22 (2015), 659-671.

[15] R. P. Agarwal, S. Hristova, D. O’Regan, Non-instantaneous Impulses in Differential Equations, Springer, Cham, 2017.

[16] E. Hernández, D. O’Regan, On a new class of abstract impulsive differential equations, Proc. Amer. Math. Soc. 141 (2013), 1641-1649.

[17] M. Pierri, D. O'Regan, V. Rolnik, Existence of solutions for semi-linear abstract differential equations with not instantaneous impulses, Appl. Math. Comput. 219 (2013), 6743-6749.

[18] D. N. Pandey, S. Das, N. Sukavanam, Existence of solution for a second-order neutral differential equation with state dependent delay and non-instantaneous impulses, Int. J. Nonlinear Sci. 18 (2014), 145-155.

[19] J. Liang, J.H. Liu, M.V. Nguyen, T.J. Xiao, Periodic mild solutions of impulsive differential equations with infinite delay in Banach spaces, J. Nonlinear Funct. Anal. 2019 (2019), Article ID 18.

[20] J.H. Liu, Periodic solutions of infinite delay evolution equations, J. Math. Anal. Appl. 247 (2000), 627-644.

[21] K. Yosida, Functional Analysis, $6^{\text {th }}$ edn., Springer-Verlag, Berlin, 1980.

[22] A. Pazy, Semigroups of Linear Operators and Applications to Partial Differential Equations, Springer-Verlag, New York, 1983.

[23] J. Hale, J. Kato, Phase space for retarded equations with infinite delay, Funkcial. Ekvac. 21 (1978), 11-41.

[24] T. Burton, Stability and Periodic Solutions of Ordinary Differential Equations and Functional Differential Equations, Academic Press, San Diego, 1985.

[25] J. Banas̀, K. Goebel, Measures of Noncompactness in Banach Spaces, Dekker, New York, 1980.

[26] K. Kuratowski, Sur les espaces complets, Fund. Math. 15 (1930), 301-309.

[27] W.A. Kirk, B. Sims, Handbook of Metric Fixed Point Theory, Springer-Science + Business Media, B.V, Dordrecht, 2001.

[28] D.J. Guo, V. Lakshmikantham, X. Liu, Nonlinear Integral Equations in Abstract Spaces, Kluwer, Dordrecht, 1996.

[29] D. Bothe, Multivalued perturbation of m-accretive differential inclusions, Isreal J. Math. 108 (1998), 109-138.

[30] H. Mönch, Boundary value problems for nonlinear ordinary differential equations of second order in Banach spaces, Nonlinear Anal. 4 (1980), 985-999.

[31] K. Goebel, Concise Course on Fixed Point Theorems, Yokohama Publishers, Japan, 2002.

[32] A. Granas, J. Dugundji, Fixed Point Theory, Springer-Verlag, New York, 2003.

[33] A. Freidman, Partial Differential Equations, Holt, Rinehart and Winston, New York, 1969. 